International Journal of Pure and Applied Mathematics

Volume 115 No. $1 \quad 2017,129-152$

ISSN: 1311-8080 (printed version); ISSN: 1314-3395 (on-line version)

url: http://www.ijpam.eu

doi: 10.12732/ijpam.v115i1.11

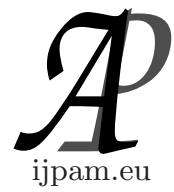

\title{
$M$-POLYNOMIALS AND TOPOLOGICAL INDICES OF SILICATE AND OXIDE NETWORKS
}

\author{
Muhammad Javaid ${ }^{1}$, Chahn Yong Jung ${ }^{2} \S$ \\ ${ }^{1}$ Department of Mathematics \\ Government College University \\ Lahore, 54000, PAKISTAN \\ ${ }^{2}$ Department of Business Administration \\ Gyeongsang National University \\ Jinju, 52828, KOREA
}

\begin{abstract}
A topological index is a numeric quantity that characterizes the whole structure of a molecular graph of the chemical compound and helps to understand its physical features, chemical reactivities and boiling activities. In 1936, Pólya introduced the concept of a counting polynomial in chemistry and Wiener in 1947 made the use of a topological index working on the boiling point of paraffin. The literature on the counting polynomials and the topological indices of the molecular graphs has grown enormously since those times. In this paper, we study the $M$-polynomials of the silicate, chain silicate and oxide networks and use these polynomials as a latest developed tool to compute the certain degree-based topological indices such as first Zagreb, second Zagreb, second modified Zagreb, general Randić, reciprocal general Randić, symmetric division deg, harmonic, inverse sum and the augmented Zagreb. we also include a comparison between all the obtained results to show the better one.
\end{abstract}

AMS Subject Classification: 05C07, 92E10

Key Words: $M$-polynomials, Zagreb indices, silicate network, oxide network

\section{Introduction}

For undefined terms, see next section. A number, polynomial or a matrix can

Received: $\quad$ May 8, 2017

Revised: June 18, 2017

Published: June 28, 2017

${ }^{\S}$ Correspondence author (c) 2017 Academic Publications, Ltd. url: www.acadpubl.eu 
uniquely identify a graph. A topological index is a function Top from $\sum$ to the set of real numbers, where $\sum$ is the set of finite simple graphs. In the progressive study of the topological indices for the molecular graphs in the chemical graph theory, many topological indices have been introduced. It is very important to know that all the topological indices are invariant under the graph theoretic operation of graphs isomorphism. The computed topological indices of the molecular graphs help to understand the physical features, chemical reactivities and boiling activities such as heat of evaporation, heat of formation, surface tension, chromatographic retention times, vapor pressure and boiling point of the involved chemical compound [5, 16, 22]. Moreover, in the studies of quantitative structure-activity relationship (QSAR) and quantitative structure-property relationship (QSPR), topological indices are utilized to guess the bioactivity of the chemical compounds [11].

In 1947, for the first time, Wiener [25] introduced the distance-based topological index in chemistry working on the boiling point of paraffin. The most studied indices are degree-based topological indices which are obtained from the degrees of the vertices of the molecular graphs under the certain formulaic conditions. This fact is emphasized in the recent survey [10] that provide a uniform approach to the degree-based topological indices.

In particular, in 1972, Gutman and Trinajsti [12] derived a pair of degreebased molecular descriptors known as the first Zagreb index and the second Zagreb index for the total $\pi$-energy of conjugated molecules. Soon after, these indices have been used as the branching indices [7, 8]. The Zagreb indices are also used in the studies of the quantitative structure-activity relationship and the quantitative structure-property relationship [11]. In 1975, Randić [21] defined the degree based topological index which is called by Randić index. In 1998, Bollobás and Erdös [4] and, in 1998, Amić et al. [1] independently defined the generalized Randić index. In 2010, the augmented Zagreb index is defined by Furtula et al. [9].

Many computational results are also obtained related to the aforesaid topological indices on different chemical structures. In particular, Rajan et al. [20] computed the certain topological indices of the silicate, honeycomb and hexagonal networks. Bača et al. [2,3] proved the topological indices for the fullerenes and the carbon nanotube networks. The rhombus type silicate and oxide networks for several topological indices are studied in [15] and the Zagreb indices of the Titania nanotubes can be found in [17]. For further study, we refer $[7,8,10,11]$.

In 1935, Pólya [19] defined the concept of counting polynomial in chemistry. Later on, in the literature, numerous polynomials are introduced with remark- 
able applications in mathematical chemistry. In particular, Hosoya polynomial is a key polynomial in the area of distance-based topological indices. The Wiener index can be obtained as the first derivative of the Hosoya polynomial at numeric value 1. Similarly, the hyper-Wiener index and the TratchStankevich-Zefirov can be computed from the Hosoya polynomial. Recently, in 2015, Deutsch and Klavzar [6] introduced the concept of $M$-polynomial and showed that the role of this polynomial for the degree-based topological indices is parallel to the role of the Hosoya polynomial for the distance-based topological indices. The $M$-polynomials and the certain degree-based topological indices of the polyhex nanotubes are studied in [18].

In this paper, we prove the $M$-polynomials of silicate, chain silicate and oxide networks. The degree-based topological indices such as first Zagreb $\left(M_{1}\right)$, second Zagreb $\left(M_{2}\right)$, second modified Zagreb $\left(M M_{2}\right)$, general Randić $\left(R_{\alpha}\right)$, reciprocal general Randić $\left(R R_{\alpha}\right)$ and symmetric division deg $(S D D)$ are computed with the help of these $M$-polynomials. In addition, we compute harmonic index $(H)$, inverse sum index $(I S)$ and the augmented Zagreb index $(A Z I)$ for all the aforesaid networks. For the better under standing a comparison between all the computed indices is also shown.

The rest of the paper is organized as: Section 2 includes the definitions and formulas which are frequently used in the main results. In Section 3, we compute the main results related to the $M$-polynomials and the certain degreebased topological indices of the silicate, chain silicate and oxide networks and, finally, Section 4 includes the conclusion between all the obtained results.

\section{Preliminaries}

A molecular graph $\Gamma=(V(\Gamma), E(\Gamma))$ with the vertex set $V(\Gamma)=\left\{v_{1}, v_{2}, \ldots, v_{n}\right\}$ and the edge set $E(\Gamma)$ is a graph whose vertices (nodes) denote atoms and edges denote bonds between the atoms of any underlying chemical structure. The order and the size of a graph are $|V(\Gamma)|=v$ and $|E(\Gamma)|=e$, respectively. A graph is connected if there exists a connection between any pair of vertices. The distance between two vertices $u$ and $v$, denoted by $d(u, v)$, is length of the shortest path between $u$ and $v$ in the graph $\Gamma$. The degree of a vertex $v$, denoted by $d(v)$, is the number of the vertices that are connected to $v$ by the edges. A loop is an edge that connects a vertex to itself, and two or more than two edges with the same end points are called multiple edges. In the present study, a molecular graph is simply a finite connected graph without multiple edges and loops. The notions and terminologies of the graphs which are used 
in this paper are standard. For further study, we refer [14, 24].

Now, we define some degree-based topological indices and polynomials which are studied in this paper.

Definition 2.1. Let $\Gamma$ be a molecular graph. Then, the first Zagreb and the second Zagreb indices denoted by $M_{1}(\Gamma)$ and $M_{2}(\Gamma)$, respectively are defined as

$$
M_{1}(\Gamma)=\sum_{u \in V(\Gamma)}[d(u)]^{2}=\sum_{u v \in E(\Gamma)}[d(u)+d(v)]
$$

and

$$
M_{2}(\Gamma)=\sum_{u v \in E(\Gamma)}[d(u) \times d(v)] .
$$

Definition 2.2. Let $\Gamma$ be a molecular graph. Then, for a real number $\alpha$, the general Randić index denoted by $R_{\alpha}(\Gamma)$ is defined as

$$
R_{\alpha}(\Gamma)=\sum_{u v \in E(\Gamma)}[d(u) \times d(v)]^{\alpha} .
$$

Definition 2.3. Let $\Gamma$ be a molecular graph. Then, the symmetry division deg index denoted by $S D D(\Gamma)$ is defined as

$$
S D D(\Gamma)=\sum_{u v \in E(\Gamma)}\left[\frac{\min (d(u), d(v))}{\max (d(u), d(v))}+\frac{\max (d(u), d(v))}{\min (d(u), d(v))}\right] .
$$

Definition 2.4. Let $\Gamma$ be a molecular graph. Then, the harmonic index and the inverse sum index of $\Gamma$ are defined as follows

$$
H(\Gamma)=\sum_{u v \in E(\Gamma)} \frac{2}{d(u)+d(v)} \quad \text { and } \quad I S(\Gamma)=\sum_{u v \in E(\Gamma)} \frac{d(u) d(v)}{d(u)+d(v)} .
$$

Definition 2.5. Let $\Gamma$ be a molecular graph. Then, augmented Zagreb index of $\Gamma$ is given by

$$
A Z I(\Gamma)=\sum_{u v \in E(\Gamma)}\left(\frac{d(u) d(v)}{d(u)+d(v)-2}\right)^{3} .
$$

Definition 2.6. Let $\Gamma$ be a molecular graph and $m_{i, j}(\Gamma) ; i, j \geq 1$ be the number of edges $e=u v$ of $\Gamma$ such that $\{d(u), d(v)\}=\{i, j\}$. Then, the $M$-polynomial of $\Gamma$ is defined as

$$
M(\Gamma, x, y)=\sum_{i \leq j}\left[m_{i, j}(\Gamma) x^{i} x^{j}\right] .
$$


In the following table, the relations between the aforesaid topological indices and the $M$-polynomial are defined.

Table 1. Derivation of degree-based topological indices from $M$-polynomial

\begin{tabular}{|c|c|l|}
\hline Indices & $f(x, y)$ & Derivation from $M(\Gamma, x, y)$ \\
\hline \hline$M_{1}$ & $x+y$ & $\left.\left(D_{x}+D_{y}\right)(M(\Gamma, x, y))\right|_{x=1=y}$ \\
\hline$M_{2}$ & $x y$ & $\left.\left(D_{x} D_{y}\right)(M(\Gamma, x, y))\right|_{x=1=y}$ \\
\hline$M M_{2}$ & $\frac{1}{x y}$ & $\left.\left(S_{x} S_{y}\right)(M(\Gamma, x, y))\right|_{x=1=y}$ \\
\hline$R_{\alpha}$ & $(x y)^{\alpha}, \alpha \in N$ & $\left.\left(D_{x}^{\alpha} D_{y}^{\alpha}\right)(M(\Gamma, x, y))\right|_{x=1=y}$ \\
\hline$R R_{\alpha}$ & $\frac{1}{(x y)^{\alpha}}, \alpha \in N$ & $\left.\left(S_{x}^{\alpha} S_{y}^{\alpha}\right)(M(\Gamma, x, y))\right|_{x=1=y}$ \\
\hline$S D D$ & $\frac{x^{2}+y^{2}}{x y}$ & $\left.\left(D_{x} S_{y}+D_{y} S_{x}\right)(M(\Gamma, x, y))\right|_{x=1=y}$ \\
\hline
\end{tabular}

Table 2. Some more degree-based topological indices from $M$-polynomial

\begin{tabular}{|c|c|l|}
\hline Indices & $f(x, y)$ & Derivation from $M(\Gamma, x, y)$ \\
\hline \hline$H$ & $\frac{2}{x+y}$ & $\left.2 S_{x} J(M(\Gamma, x, y))\right|_{x=1}$ \\
\hline$I S$ & $\frac{x y}{x+y}$ & $\left.S_{x} Q_{2} J D_{x} D_{y}(M(\Gamma, x, y))\right|_{x=1}$ \\
\hline$A Z I$ & $\left(\frac{x y}{x+y-2}\right)^{3}$ & $\left.S_{x}^{3} J D_{x}^{3} D_{y}^{3}(M(\Gamma, x, y))\right|_{x=1}$ \\
\hline
\end{tabular}

In the Table $1, M M_{2}$ is second modified Zagreb, $R R_{\alpha}$ is reciprocal general Randić, $D_{x}=\frac{\partial(f(x, y))}{\partial(x)}, D_{y}=\frac{\partial(f(x, y))}{\partial(y)}, S_{x}=\int_{0}^{x} \frac{f(t, y)}{t} d t$ and $S_{y}=\int_{0}^{y} \frac{f(x, t)}{t} d t$.

In the Table $2, J(f(x, y))=f(x, x)$ and $Q_{\alpha}(f(x, y))=x^{\alpha} f(x, y)$, where $\alpha \neq 0$. To know in details for these operators, we refer [6].

Now, we discuss the construction of silicate, chain silicate and oxide networks. The class of the silicates is a most interesting class of minerals by far which are obtained by fusing metal oxides or metal carbonates with sand. Essentially all the silicates contain $\mathrm{SiO}_{4}$ tetrahedra as its basic unit. In chemistry, the corner vertices of $\mathrm{SiO}_{4}$ tetrahedron represent oxygen ions and the central vertex represents the silicon ion. In graph theory, we call the corner vertices as oxygen nodes and the center vertex as silicon node. By the different arrangements of the tetrahedron silicate, we obtain different silicate structures. Similarly, different silicate networks are constructed by the different silicate structures.

In Figure 1, the silicate network of dimension 2 is presented. In general, the vertices and the edges in a silicate network $S L(n)$ of dimension $n$ are $|V(S L(n))|=15 n^{2}+3 n$ and $|E(S L(n))|=36 n^{2}$, respectively. A chain silicate network denoted by $C S(n)$ is obtained by arranging $n$ tetrahedra linearly. The Figure 2 presents the chain silicate network for $n=6$. The vertices and edges of 
the chain silicate network $C S(n)$ are $|V(C S(n))|=3 n+1$ and $|E(C S(n))|=6 n$. Moreover, If we delete all the silicon ions from the silicate network, then we obtain oxide network as shown in Figure 3 of dimension 2. For dimension $n$, the vertices and the edges of a oxide network $O X(n)$ are $|V(O X(n))|=9 n^{2}+3 n$ and $|E(O X(n))|=18 n^{2}$, respectively.

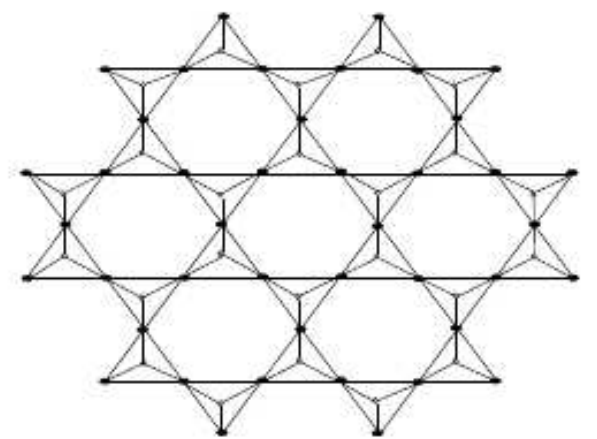

Figure 1. Silicate network for $n=2(S L(2))$ with solid vertices as oxygen atoms and plain vertices as silicate atoms

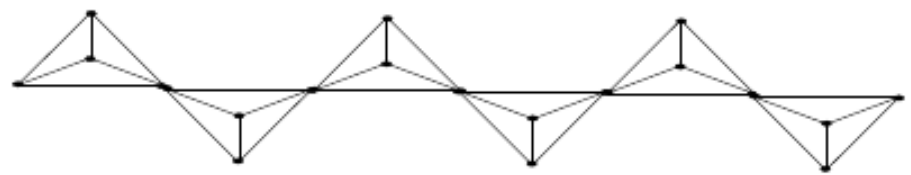

Figure 2. Chain silicate network for $n=6(C S(6))$

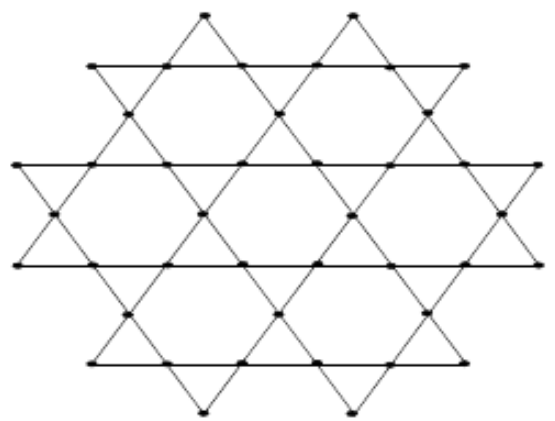

Figure 3. Oxide network for $n=2(O X(2))$ 


\section{Main Results}

In this section, we present the main results related to the $M$-polynomials and the certain degree-based topological indices of the silicate, chain silicate and oxide networks.

Theorem 3.1. Let $\Gamma=S L(n)$ be the silicate network. Then, the $M$ polynomial of $\Gamma$ is

$$
M(\Gamma, x, y)=(6 n) x^{3} y^{3}+\left(18 n^{2}+6 n\right) x^{3} y^{6}+\left(18 n^{2}-12 n\right) x^{6} y^{6} .
$$

Proof. From Figure 1, we note that there are two types of vertices in $\Gamma$ with respect to their degree such as of degree 3 and 6 , and three types of edges with respect to degree of end vertices, that is, $\{3,3\},\{3,6\}$ and $\{6,6\}$. Thus, we have

$$
V_{1}=\{u \in V(\Gamma) \mid d(u)=3\} \quad \text { and } \quad V_{2}=\{u \in V(\Gamma) \mid d(u)=6\}
$$

with $\left|V_{1}\right|=6 n^{2}+6 n$, and $\left|V_{2}\right|=9 n^{2}-3 n$, respectively. Consequently, $|V(\Gamma)|=$ $15 n^{2}+3 n$. Similarly, we have

$$
\begin{aligned}
& E_{1}=E_{3,3}=\{u v \in E(\Gamma) \mid d(u)=3, d(u)=3\} \\
& E_{2}=E_{3,6}=\{u v \in E(\Gamma) \mid d(u)=3, d(u)=6\} \\
& E_{3}=E_{6,6}=\{u v \in E(\Gamma) \mid d(u)=6, d(u)=6\}
\end{aligned}
$$

such that $\left|E_{1}\right|=6 n,\left|E_{2}\right|=18 n^{2}+6 n$ and $\left|E_{3}\right|=18 n^{2}-12 n$. We conclude $|E(\Gamma)|=36 n^{2}$. Thus, the partitions of the vertex set and the edge set of the silicate network are given in the Tables 3 and 4 .

Table 3. The partitions of the vertex set of the silicate network $S L(n)$

\begin{tabular}{|c|c|c|}
\hline Vertex partition & $V_{1}$ & $V_{2}$ \\
\hline Cardinality & $6 n^{2}+6 n$ & $9 n^{2}-3 n$ \\
\hline
\end{tabular}

Table 4. The partitions of the edge set of the silicate network $S L(n)$

\begin{tabular}{|c|c|c|c|}
\hline Edge partition & $E_{1}=E_{3,3}$ & $E_{2}=E_{3,6}$ & $E_{3}=E_{6,6}$ \\
\hline Cardinality & $6 n$ & $18 n^{2}+6 n$ & $18 n^{2}-12 n$ \\
\hline
\end{tabular}

Now, by the use of Definition 2.6 and the Tables 2 and 3, $M$-polynomial of 
$\Gamma$ is

$$
\begin{aligned}
M(\Gamma, x, y) & =\sum_{i \leq j}\left[E_{i, j}(\Gamma) x^{i} y^{j}\right] \\
& =\sum_{3 \leq 3}\left[E_{3,3}(\Gamma) x^{3} y^{3}\right]+\sum_{3 \leq 6}\left[E_{3,6}(\Gamma) x^{3} y^{6}\right]+\sum_{6 \leq 6}\left[E_{6,6}(\Gamma) x^{6} y^{6}\right] \\
& =\left|E_{1}\right| x^{3} y^{3}+\left|E_{2}\right| x^{3} y^{6}+\left|E_{3}\right| x^{6} y^{6} \\
& =(6 n) x^{3} y^{3}+\left(18 n^{2}+6 n\right) x^{3} y^{6}+\left(18 n^{2}-12 n\right) x^{6} y^{6}
\end{aligned}
$$

Theorem 3.2. Let $\Gamma=S L(n)$ be the silicate network and

$$
M(\Gamma, x, y)=6 n x^{3} y^{3}+\left(18 n^{2}+6 n\right) x^{3} y^{6}+\left(18 n^{2}-12 n\right) x^{6} y^{6}
$$

be its $M$-polynomial. Then, the first Zagreb index $\left(M_{1}(\Gamma)\right)$, the second Zagreb index $\left(M_{2}(\Gamma)\right)$, the second modified Zagreb $\left(M M_{2}(\Gamma)\right)$, general Randić, $\left(R_{\alpha}(\Gamma)\right)$, where $\alpha \in \mathbb{N}$, reciprocal general Randić, $\left(R R_{\alpha}(\Gamma)\right)$, where $\alpha \in \mathbb{N}$ and the symmetric division degree index $(S D D(\Gamma))$ obtained from $M$-polynomial are as follows:
(a) $M_{1}(\Gamma)=378 n^{2}-54 n$,
(b) $M_{2}(\Gamma)=972 n^{2}-270 n$,
(c) $M M_{2}(\Gamma)=\frac{1}{6}\left[9 n^{2}+4 n\right]$,
(d) $R_{\alpha}(\Gamma)=(3)^{2 \alpha}\left[2^{\alpha}\left(1+2^{\alpha}\right)\left(18 n^{2}\right)+\left(2^{\alpha}\left(1-2^{\alpha+1}\right)+1\right)(6 n)\right]$,
(e) $R R_{\alpha}(\Gamma)=\frac{1}{(6)^{2 \alpha-1}}\left[\left(2^{\alpha}+1\right)\left(3 n^{2}\right)+\left(2^{2 \alpha}+2^{\alpha}-2\right) n\right]$,
(f) $S D D(\Gamma)=81 n^{2}+3 n$.

Proof. Let $f(x, y)=M(\Gamma, x, y)$ be the $M$-polynomial of the silicate network. Then

$$
f(x, y)=(6 n) x^{3} y^{3}+\left(18 n^{2}+6 n\right) x^{3} y^{6}+\left(18 n^{2}-12 n\right) x^{6} y^{6} .
$$

Now, the required partial derivatives and integrals are obtained as $D_{x}(f(x, y))=18 n x^{2} y^{3}+3\left(18 n^{2}+6 n\right) x^{2} y^{6}+6\left(18 n^{2}-12 n\right) x^{5} y^{6}$, $D_{y}(f(x, y))=18 n x^{3} y^{2}+6\left(18 n^{2}+6 n\right) x^{3} y^{5}+6\left(18 n^{2}-12 n\right) x^{6} y^{5}$, $D_{x}\left(D_{y}(f(x, y))\right)=54 n x^{2} y^{2}+18\left(18 n^{2}+6 n\right) x^{2} y^{5}+36\left(18 n^{2}-12 n\right) x^{5} y^{5}$, $S_{x}(f(x, y))=2 n x^{3} y^{3}+\left(6 n^{2}+2 n\right) x^{3} y^{6}+\left(3 n^{2}-2 n\right) x^{6} y^{6}$, $S_{y}(f(x, y))=2 n x^{3} y^{3}+\left(3 n^{2}+n\right) x^{3} y^{6}+\left(3 n^{2}-2 n\right) x^{6} y^{6}$, $S_{x} S_{y}(f(x, y))=\frac{2}{3}(n) x^{3} y^{3}+\frac{1}{3}\left(3 n^{2}+n\right) x^{3} y^{6}+\frac{1}{6}\left(3 n^{2}-2 n\right) x^{6} y^{6}$, $D_{y} S_{x}(f(x, y))=6 n x^{3} y^{2}+\left(36 n^{2}+12 n\right) x^{3} y^{5}+\left(18 n^{2}-12 n\right) x^{6} y^{5}$, $D_{x} S_{y}(f(x, y))=6 n x^{2} y^{3}+\left(9 n^{2}+3 n\right) x^{2} y^{6}+\left(18 n^{2}-12 n\right) x^{5} y^{6}$, 
$D_{x}^{\alpha}\left(D_{y}^{\alpha}(f(x, y))\right)=(9)^{\alpha}(6 n) x^{2} y^{2}+(18)^{\alpha}\left(18 n^{2}+6 n\right) x^{2} y^{5}+(36)^{\alpha}\left(18 n^{2}-\right.$ $12 n) x^{5} y^{5}$

$S_{x}^{\alpha} S_{y}^{\alpha}(f(x, y))=\left(\frac{6 n}{9^{\alpha}}\right) x^{3} y^{3}+\left(\frac{18 n^{2}+6 n}{18^{\alpha}}\right) x^{3} y^{6}+\left(\frac{18 n^{2}-12 n}{36^{\alpha}}\right) x^{6} y^{6}$.

Now, we obtain

$\left.D_{x}(f(x, y))\right|_{x=1=y}=162 n^{2}-36 n$,

$\left.D_{y}(f(x, y))\right|_{x=1=y}=216 n^{2}-18 n$,

$\left.D_{x}\left(D_{y}(f(x, y))\right)\right|_{x=1=y}=972 n^{2}-270 n$,

$\left.S_{x}(f(x, y))\right|_{x=1=y}=9 n^{2}+2 n$,

$\left.S_{y}(f(x, y))\right|_{x=1=y}=6 n^{2}+n$,

$\left.S_{x} S_{y}(f(x, y))\right|_{x=1=y}=\frac{2 n}{3}+\frac{3 n^{2}+n}{3}+\frac{\left.3 n^{2}-2 n\right)}{6}$,

$\left.D_{y} S_{x}(f(x, y))\right|_{x=1=y}=54 n^{2}+6 n$,

$\left.D_{x} S_{y}(f(x, y))\right|_{x=1=y}=27 n^{2}-3 n$,

$\left.D_{x}^{\alpha}\left(D_{y}^{\alpha}(f(x, y))\right)\right|_{x=1=y}=(9)^{\alpha}(6 n)+(18)^{\alpha}\left(18 n^{2}+6 n\right)+(36)^{\alpha}\left(18 n^{2}-12 n\right)$,

$\left.S_{x}^{\alpha} S_{y}^{\alpha}(f(x, y))\right|_{x=1=y}=\frac{6 n}{9^{\alpha}}+\frac{18 n^{2}+6 n}{18^{\alpha}}+\frac{18 n^{2}-12 n}{36^{\alpha}}$.

Consequently,

(a)

$$
\begin{aligned}
M_{1}(\Gamma) & =\left.\left(D_{x}+D_{y}\right)(f(x, y))\right|_{x=1=y} \\
& =\left.D_{x}(f(x, y))\right|_{x=1=y}+\left.D_{y}(f(x, y))\right|_{x=1=y} \\
& =\left(162 n^{2}-36 n\right)+\left(216 n^{2}-18 n\right)=378 n^{2}-54 n
\end{aligned}
$$

(b)

$$
\begin{aligned}
M_{2}(\Gamma) & =\left.\left(D_{x} D_{y}\right)(f(x, y))\right|_{x=1=y} \\
& =\left.D_{x}\left(D_{y}(f(x, y))\right)\right|_{x=1=y}=972 n^{2}-270 n
\end{aligned}
$$

(c)

$$
\begin{aligned}
M M_{2}(\Gamma) & =\left.\left(S_{x} S_{y}\right)(f(x, y))\right|_{x=1=y} \\
& =\left.S_{x}\left(S_{y}(f(x, y))\right)\right|_{x=1=y} \\
& =\frac{2 n}{3}+\frac{3 n^{2}+n}{3}+\frac{3 n^{2}-2 n}{6}=\frac{1}{6}\left[9 n^{2}+4 n\right],
\end{aligned}
$$

$(d)$

$$
\begin{aligned}
R_{\alpha}(\Gamma) & =\left.\left(D_{x}^{\alpha} D_{y}^{\alpha}\right)(f(x, y))\right|_{x=1=y} \\
& =(9)^{\alpha}(6 n)+(18)^{\alpha}\left(18 n^{2}+6 n\right)+(36)^{\alpha}\left(18 n^{2}-12 n\right) \\
& =(3)^{2 \alpha}\left[2^{\alpha}\left(1+2^{\alpha}\right)\left(18 n^{2}\right)+\left(2^{\alpha}\left(1-2^{\alpha+1}\right)+1\right)(6 n)\right]
\end{aligned}
$$


(e)

$$
\begin{aligned}
R R_{\alpha}(\Gamma) & =\left.\left(S_{x}^{\alpha} S_{y}^{\alpha}\right)(f(x, y))\right|_{x=1=y} \\
& =\frac{6 n}{9^{\alpha}}+\frac{18 n^{2}+6 n}{18^{\alpha}}+\frac{18 n^{2}-12 n}{36^{\alpha}} \\
& =\frac{1}{(6)^{2 \alpha-1}}\left[\left(2^{\alpha}+1\right)\left(3 n^{2}\right)+\left(2^{2 \alpha}+2^{\alpha}-2\right) n\right]
\end{aligned}
$$

$(f)$

$$
\begin{aligned}
S D D(\Gamma) & =\left(D_{x} S_{y}+D_{y} S_{x}\right)(f(x, y)) \\
& =\left(D_{x} S_{y}\right)(f(x, y))+\left(D_{y} S_{x}\right)(f(x, y)) \\
& =D_{x}\left(S_{y}(f(x, y))\right)+D_{y}\left(S_{x}(f(x, y))\right) \\
& =\left(54 n^{2}+6 n\right)+\left(27 n^{2}-3 n\right) \\
& =81 n^{2}+3 n .
\end{aligned}
$$

Theorem 3.3. Let $\Gamma=S L(n)$ be the silicate network and

$$
M(\Gamma, x, y)=6 n x^{3} y^{3}+\left(18 n^{2}+6 n\right) x^{3} y^{6}+\left(18 n^{2}-12 n\right) x^{6} y^{6}
$$

be its $M$-polynomial. Then, harmonic index $(H(\Gamma))$, inverse sum index $(I S(\Gamma))$, and augmented Zagreb index $(A Z I(\Gamma))$ obtained from $M$-polynomial are as follows:

(a) $H(\Gamma)=\frac{n}{3}[21 n+4]$,

(b) $I S(\Gamma)=15 n(6 n-1)$,

(c) $A Z I(\Gamma)=\frac{49128768}{42875} n^{2}-\frac{534408759}{1372000} n$.

Proof. Let $f(x, y)=M(\Gamma, x, y)$ be the $M$-polynomial of the silicate network. Then

$$
f(x, y)=6 n x^{3} y^{3}+\left(18 n^{2}+6 n\right) x^{3} y^{6}+\left(18 n^{2}-12 n\right) x^{6} y^{6} .
$$

Now, the required expressions are obtained as

$J(f(x, y))=6 n x^{6}+\left(18 n^{2}+6 n\right) x^{9}+\left(18 n^{2}-12 n\right) x^{12}$,

$S_{x}(J f(x, y))=n x^{6}+\frac{6 n^{2}+2 n}{3} x^{9}+\frac{3 n^{2}-2 n}{2} x^{12}$,

$J\left(D_{x}\left(D_{y}(f(x, y))\right)\right)=54 n x^{4}+18\left(18 n^{2}+6 n\right) x^{7}+36\left(18 n^{2}-12 n\right) x^{10}$,

$Q_{2} J\left(D_{x}\left(D_{y}(f(x, y))\right)\right)=54 n x^{6}+18\left(18 n^{2}+6 n\right) x^{9}+36\left(18 n^{2}-12 n\right) x^{12}$,

$S_{x} Q_{2} J\left(D_{x}\left(D_{y}(f(x, y))\right)\right)=9 n x^{6}+2\left(18 n^{2}+6 n\right) x^{9}+3\left(18 n^{2}-12 n\right) x^{12}$,

$D_{x}^{3}\left(D_{y}^{3}(f(x, y))\right)=(9)^{3}(6 n) x^{2} y^{2}+(18)^{3}\left(18 n^{2}+6 n\right) x^{2} y^{5}+(36)^{3}\left(18 n^{2}-\right.$ $12 n) x^{5} y^{5}$

$J D_{x}^{3}\left(D_{y}^{3}(f(x, y))\right)=(9)^{3}(6 n) x^{4}+(18)^{3}\left(18 n^{2}+6 n\right) x^{7}+(36)^{3}\left(18 n^{2}-12 n\right) x^{10}$, 
$S_{x}^{3} J\left(D_{x}^{3}\left(D_{y}^{3}(f(x, y))\right)\right)=6 n\left(\frac{9}{4}\right)^{3} x^{4}+\left(\frac{18}{7}\right)^{3}\left(18 n^{2}+6 n\right) x^{7}+\left(\frac{36}{10}\right)^{3}\left(18 n^{2}-\right.$ $12 n) x^{10}$.

Now, we obtain

$\left.S_{x}(J f(x, y))\right|_{x=1=y}=n+\frac{6 n^{2}+2 n}{3}+\frac{3 n^{2}-2 n}{2}$,

$\left.S_{x} Q_{2} J\left(D_{x}\left(D_{y}(f(x, y))\right)\right)\right|_{x=1=y}=9 n+2\left(18 n^{2}+6 n\right)+3\left(18 n^{2}-12 n\right)$,

$\left.S_{x}^{3} J\left(D_{x}^{3}\left(D_{y}^{3}(f(x, y))\right)\right)\right|_{x=1=y}=6 n\left(\frac{9}{4}\right)^{3}+\left(\frac{18}{7}\right)^{3}\left(18 n^{2}+6 n\right)+\left(\frac{36}{10}\right)^{3}\left(18 n^{2}-\right.$ $12 n)$.

Consequently,

(a)

$$
\begin{aligned}
H(\Gamma) & =\left.2 S_{x}(J f(x, y))\right|_{x=1=y} \\
& =2\left[n+\frac{6 n^{2}+2 n}{3}+\frac{3 n^{2}-2 n}{2}\right]=\frac{n}{3}[21 n+4],
\end{aligned}
$$

(b)

$$
\begin{aligned}
I S(\Gamma) & =\left.S_{x} Q_{2} J\left(D_{x}\left(D_{y}(f(x, y))\right)\right)\right|_{x=1=y} \\
& =9 n+2\left(18 n^{2}+6 n\right)+3\left(18 n^{2}-12 n\right) \\
& =15 n(6 n-1),
\end{aligned}
$$

(c)

$$
\begin{aligned}
A Z I(\Gamma) & =\left.S_{x}^{3} J\left(D_{x}^{3}\left(D_{y}^{3}(f(x, y))\right)\right)\right|_{x=1=y} \\
& =6 n\left(\frac{9}{4}\right)^{3}+\left(\frac{18}{7}\right)^{3}\left(18 n^{2}+6 n\right)+\left(\frac{36}{10}\right)^{3}\left(18 n^{2}-12 n\right), \\
& =\frac{49128768}{42875} n^{2}-\frac{534408759}{1372000} n .
\end{aligned}
$$

Theorem 3.4. Let $\Gamma=C S(n)$ be the chain silicate network. Then, the $M$-polynomial of $\Gamma$ is

$$
M(\Gamma, x, y)=(n+4) x^{3} y^{3}+(4 n-2) x^{3} y^{6}+(n-2) x^{6} y^{6}
$$

Proof. From Figure 2, we note that there are two types of vertices in $\Gamma$ with respect to their degree such as of degree 3 and 6 , and three types of edges with respect to degree of end vertices, that is, $\{3,3\},\{3,6\}$ and $\{6,6\}$. Thus, we have

$$
V_{1}=\{u \in V(\Gamma) \mid d(u)=3\} \quad \text { and } \quad V_{2}=\{u \in V(\Gamma) \mid d(u)=6\}
$$


with $\left|V_{1}\right|=2 n+2$, and $\left|V_{2}\right|=n-1$, respectively. Consequently, $|V(\Gamma)|=3 n+1$. Similarly, we have

$$
\begin{aligned}
& E_{1}=E_{3,3}=\{u v \in E(\Gamma) \mid d(u)=3, d(u)=3\}, \\
& E_{2}=E_{3,6}=\{u v \in E(\Gamma) \mid d(u)=3, d(u)=6\}, \\
& E_{3}=E_{6,6}=\{u v \in E(\Gamma) \mid d(u)=6, d(u)=6\}
\end{aligned}
$$

such that $\left|E_{1}\right|=n+4,\left|E_{2}\right|=4 n-2$ and $\left|E_{3}\right|=n-2$, where $n \geq 2$. We conclude $|E(\Gamma)|=6 n$, for $n \geq 2$. Thus, the partitions of the vertex set and the edge set of the chain silicate network are given in the Tables 5 and 6 .

Table 5. The partitions of the vertex set of the chain silicate network $C S(n)$

\begin{tabular}{|c|c|c|}
\hline Vertex partition & $V_{1}$ & $V_{2}$ \\
\hline Cardinality & $2 n+2$ & $n-1$ \\
\hline
\end{tabular}

Table 6. The partitions of the egde set of the chain silicate network $C S(n)$

\begin{tabular}{|c|c|c|c|}
\hline Edge partition & $E_{1}=E_{3,3}$ & $E_{2}=E_{3,6}$ & $E_{3}=E_{6,6}$ \\
\hline Cardinality & $n+4$ & $4 n-2$ & $n-2$ \\
\hline
\end{tabular}

Now, by the use of Definition 2.6 and the Tables 5 and $6, M$-polynomial of $\Gamma$ is

$$
\begin{aligned}
M(\Gamma, x, y) & =\sum_{i \leq j}\left[E_{i, j}(\Gamma) x^{i} y^{j}\right] \\
& =\sum_{3 \leq 3}\left[E_{3,3}(\Gamma) x^{3} y^{3}\right]+\sum_{3 \leq 6}\left[E_{3,6}(\Gamma) x^{3} y^{6}\right]+\sum_{6 \leq 6}\left[E_{6,6}(\Gamma) x^{6} y^{6}\right] \\
& =\left|E_{1}\right| x^{3} y^{3}+\left|E_{2}\right| x^{3} y^{6}+\left|E_{3}\right| x^{6} y^{6} \\
& =(n+4) x^{3} y^{3}+(4 n-2) x^{3} y^{6}+(n-2) x^{6} y^{6} .
\end{aligned}
$$

Theorem 3.5. Let $\Gamma=C S(n)$ be the chain silicate network and

$$
M(\Gamma, x, y)=(n+4) x^{3} y^{3}+(4 n-2) x^{3} y^{6}+(n-2) x^{6} y^{6}
$$

be its $M$-polynomial. Then, the first Zagreb index $\left(M_{1}(\Gamma)\right)$, the second Zagreb index $\left(M_{2}(\Gamma)\right)$, the second modified Zagreb $\left(M M_{2}(\Gamma)\right)$, general Randic, $\left(R_{\alpha}(\Gamma)\right)$, where $\alpha \in \mathbb{N}$, reciprocal general Randić, $\left(R R_{\alpha}(\Gamma)\right)$, where $\alpha \in \mathbb{N}$ and 
the symmetric division degree index $(S D D(\Gamma))$ obtained from $M$-polynomial are as follows:
(a) $M_{1}(\Gamma)=54 n-18$,
(b) $M_{2}(\Gamma)=117 n-72$,
(c) $M M_{2}(\Gamma)=\frac{1}{36}[13 n+10]$,
(d) $R_{\alpha}(\Gamma)=(9)^{\alpha}\left[n\left(2^{2 \alpha}+2^{\alpha+2}+1\right)+2\left(2-2^{\alpha}-2^{2 \alpha}\right)\right]$,
(e) $R R_{\alpha}(\Gamma)=\frac{1}{(6)^{2 \alpha}}\left[n\left(2^{2 \alpha}+2^{\alpha+2}+1\right)+2\left(2^{2 \alpha+1}-2^{\alpha}-1\right)\right]$,
(f) $S D D(\Gamma)=14 n-1$.

Proof. Let $f(x, y)=M(\Gamma, x, y)$ be the $M$-polynomial of the chain silicate network. Then

$$
f(x, y)=(n+4) x^{3} y^{3}+(4 n-2) x^{3} y^{6}+(n-2) x^{6} y^{6} .
$$

Now, the required partial derivatives and integrals are obtained as

$$
\begin{aligned}
& D_{x}(f(x, y))=3(n+4) x^{2} y^{3}+3(4 n-2) x^{2} y^{6}+6(n-2) x^{5} y^{6}, \\
& D_{y}(f(x, y))=3(n+4) x^{3} y^{2}+6(4 n-2) x^{3} y^{5}+6(n-2) x^{6} y^{5}, \\
& D_{x}\left(D_{y}(f(x, y))\right)=9(n+4) x^{2} y^{2}+18(4 n-2) x^{2} y^{5}+36(n-2) x^{5} y^{5}, \\
& S_{x}(f(x, y))=\frac{n+4}{3} x^{3} y^{3}+\frac{4 n-2}{3} x^{3} y^{6}+\frac{n-2}{6} x^{6} y^{6}, \\
& S_{y}(f(x, y))=\frac{n+4}{3} x^{3} y^{3}+\frac{4 n-2}{6} x^{3} y^{6}+\frac{n-2}{6} x^{6} y^{6}, \\
& S_{x} S_{y}(f(x, y))=\frac{n+4}{9} x^{3} y^{3}+\frac{4 n-2}{18} x^{3} y^{6}+\frac{n-2}{36} x^{6} y^{6}, \\
& D_{y} S_{x}(f(x, y))=(n+4) x^{3} y^{2}+2(4 n-2) x^{3} y^{5}+(n-2) x^{6} y^{5}, \\
& D_{x} S_{y}(f(x, y))=(n+4) x^{2} y^{3}+(2 n-1) x^{2} y^{6}+(n-2) x^{5} y^{6}, \\
& D_{x}^{\alpha}\left(D_{y}^{\alpha}(f(x, y))\right)=\left(9^{\alpha}\right)(n+4) x^{2} y^{2}+\left(18^{\alpha}\right)(4 n-2) x^{2} y^{5}+\left(36^{\alpha}\right)(n-2) x^{5} y^{5}, \\
& S_{x}^{\alpha} S_{y}^{\alpha}(f(x, y))=\frac{n+4}{9^{\alpha}} x^{3} y^{3}+\frac{4 n-2}{18^{\alpha}} x^{3} y^{6}+\frac{n-2}{36^{\alpha}} x^{6} y^{6} .
\end{aligned}
$$

Now, we obtain

$$
\begin{aligned}
& \left.D_{x}(f(x, y))\right|_{x=1=y}=21 n-6, \\
& \left.D_{y}(f(x, y))\right|_{x=1=y}=33 n-12, \\
& \left.D_{x}\left(D_{y}(f(x, y))\right)\right|_{x=1=y}=117 n-72, \\
& \left.S_{x}(f(x, y))\right|_{x=1=y}=\frac{1}{6}[11 n+2], \\
& \left.S_{y}(f(x, y))\right|_{x=1=y}=\frac{1}{6}[7 n+4], \\
& \left.S_{x} S_{y}(f(x, y))\right|_{x=1=y}=\frac{n+4}{9}+\frac{4 n-2}{18}+\frac{n-2}{36}, \\
& \left.D_{y} S_{x}(f(x, y))\right|_{x=1=y}=10 n-2, \\
& \left.D_{x} S_{y}(f(x, y))\right|_{x=1=y}=4 n+1, \\
& \left.D_{x}^{\alpha}\left(D_{y}^{\alpha}(f(x, y))\right)\right|_{x=1=y}=\left(9^{\alpha}\right)(n+4)+\left(18^{\alpha}\right)(4 n-2)+\left(36^{\alpha}\right)(n-2), \\
& \left.S_{x}^{\alpha} S_{y}^{\alpha}(f(x, y))\right|_{x=1=y}=\frac{6 n}{9^{\alpha}}+\frac{18 n^{2}+6 n}{18^{\alpha}}+\frac{18 n^{2}-12 n}{36^{\alpha}} .
\end{aligned}
$$

Consequently, 
(a)

$$
\begin{aligned}
M_{1}(\Gamma) & =\left.\left(D_{x}+D_{y}\right)(f(x, y))\right|_{x=1=y} \\
& =\left.D_{x}(f(x, y))\right|_{x=1=y}+\left.D_{y}(f(x, y))\right|_{x=1=y} \\
& =(21 n-6)+(33 n-12)=54 n-18,
\end{aligned}
$$

(b)

$$
\begin{aligned}
M_{2}(\Gamma) & =\left.\left(D_{x} D_{y}\right)(f(x, y))\right|_{x=1=y} \\
& =\left.D_{x}\left(D_{y}(f(x, y))\right)\right|_{x=1=y}=117 n-72
\end{aligned}
$$

(c)

$$
\begin{aligned}
M M_{2}(\Gamma) & =\left.\left(S_{x} S_{y}\right)(f(x, y))\right|_{x=1=y} \\
& =\left.S_{x}\left(S_{y}(f(x, y))\right)\right|_{x=1=y} \\
& =\frac{n+4}{9}+\frac{4 n-2}{18}+\frac{n-2}{36}=\frac{1}{36}[13 n+10]
\end{aligned}
$$

(d)

$$
\begin{aligned}
R_{\alpha}(\Gamma) & =\left.\left(D_{x}^{\alpha} D_{y}^{\alpha}\right)(f(x, y))\right|_{x=1=y} \\
& =\left(9^{\alpha}\right)(n+4)+\left(18^{\alpha}\right)(4 n-2)+\left(36^{\alpha}\right)(n-2) \\
& =(9)^{\alpha}\left[n\left(2^{2 \alpha}+2^{\alpha+2}+1\right)+2\left(2-2^{\alpha}-2^{2 \alpha}\right)\right],
\end{aligned}
$$

(e)

$$
\begin{aligned}
R R_{\alpha}(\Gamma) & =\left.\left(S_{x}^{\alpha} S_{y}^{\alpha}\right)(f(x, y))\right|_{x=1=y} \\
& =\frac{6 n}{9^{\alpha}}+\frac{18 n^{2}+6 n}{18^{\alpha}}+\frac{18 n^{2}-12 n}{36^{\alpha}} \\
& =\frac{1}{(6)^{2 \alpha}}\left[n\left(2^{2 \alpha}+2^{\alpha+2}+1\right)+2\left(2^{2 \alpha+1}-2^{\alpha}-1\right)\right]
\end{aligned}
$$

$(f)$

$$
\begin{aligned}
S D D(\Gamma) & =\left(D_{x} S_{y}+D_{y} S_{x}\right)(f(x, y)) \\
& =\left(D_{x} S_{y}\right)(f(x, y))+\left(D_{y} S_{x}\right)(f(x, y)) \\
& =D_{x}\left(S_{y}(f(x, y))\right)+D_{y}\left(S_{x}(f(x, y))\right) \\
& =(10 n-2)+(4 n+1)=14 n-1 .
\end{aligned}
$$

Theorem 3.6. Let $\Gamma=C S(n)$ be the silicate network and

$$
M(\Gamma, x, y)=(n+4) x^{3} y^{3}+(4 n-2) x^{3} y^{6}+(n-2) x^{6} y^{6}
$$


be its $M$-polynomial. Then, harmonic index $(H(\Gamma))$, inverse sum index $(I S(\Gamma))$, and augmented Zagreb index $(A Z I(\Gamma))$ obtained from $M$-polynomial are as follows:

(a) $H(\Gamma)=\frac{1}{18}[21 n+10]$,

(b) $I S(\Gamma)=2(7 n-2)$,

(c) $A Z I(\Gamma)=\frac{345903939}{2744000} n-\frac{56084157}{686000}$.

Proof. Let $f(x, y)=M(\Gamma, x, y)$ be the $M$-polynomial of the chain silicate network. Then

$$
f(x, y)=(n+4) x^{3} y^{3}+(4 n-2) x^{3} y^{6}+(n-2) x^{6} y^{6} .
$$

Now, the required expressions are obtained as

$J(f(x, y))=(n+4) x^{6}+(4 n-2) x^{9}+(n-2) x^{12}$,

$S_{x}(J f(x, y))=\frac{n+4}{6} x^{6}+\frac{4 n-2}{9} x^{9}+\frac{n-2}{12} x^{12}$,

$J\left(D_{x}\left(D_{y}(f(x, y))\right)\right)=9(n+4) x^{4}+18(4 n-2) x^{7}+36(n-2) x^{10}$,

$Q_{2} J\left(D_{x}\left(D_{y}(f(x, y))\right)\right)=9(n+4) x^{6}+18(4 n-2) x^{9}+36(n-2) x^{12}$,

$S_{x} Q_{2} J\left(D_{x}\left(D_{y}(f(x, y))\right)\right)=\frac{9}{6}(n+4) x^{6}+\frac{18}{9}(4 n-2) x^{9}+\frac{36}{12}(n-2) x^{12}$,

$D_{x}^{3}\left(D_{y}^{3}(f(x, y))\right)=\left(9^{3}\right)(n+4) x^{2} y^{2}+\left(18^{3}\right)(4 n-2) x^{3} y^{6}+\left(36^{3}\right)(n-2) x^{6} y^{6}$,

$J D_{x}^{3}\left(D_{y}^{3}(f(x, y))\right)=\left(9^{3}\right)(n+4) x^{6}+\left(18^{3}\right)(4 n-2) x^{9}+\left(36^{3}\right)(n-2) x^{12}$,

$S_{x}^{3} J\left(D_{x}^{3}\left(D_{y}^{3}(f(x, y))\right)\right)=\left(\frac{9}{4}\right)^{3}(n+4) x^{6}+\left(\frac{18}{7}\right)^{3}(4 n-2) x^{9}+\left(\frac{36}{10}\right)^{3}(n-2) x^{12}$.

Now, we obtain

$\left.S_{x}(J f(x, y))\right|_{x=1=y}=\frac{n+4}{6}+\frac{4 n-2}{9}+\frac{n-2}{12}$,

$\left.S_{x} Q_{2} J\left(D_{x}\left(D_{y}(f(x, y))\right)\right)\right|_{x=1=y}=\frac{9(n+4)}{6}+\frac{18(4 n-2)}{9}+\frac{(n-2)}{12}$,

$\left.S_{x}^{3} J\left(D_{x}^{3}\left(D_{y}^{3}(f(x, y))\right)\right)\right|_{x=1=y}=\left(\frac{9(n+4)}{4}\right)^{3}+\left(\frac{18(4 n-2)}{7}\right)^{3}+\left(\frac{36(n-2)}{10}\right)^{3}$.

Consequently,

(a)

$$
\begin{aligned}
H(\Gamma) & =\left.2 S_{x}(J f(x, y))\right|_{x=1=y} \\
& =2\left[\frac{n+4}{6}+\frac{4 n-2}{9}+\frac{n-2}{12}\right]=\frac{1}{18}[21 n+10]
\end{aligned}
$$

(b)

$$
\begin{aligned}
I S(\Gamma) & =\left.S_{x} Q_{2} J\left(D_{x}\left(D_{y}(f(x, y))\right)\right)\right|_{x=1=y} \\
& =\frac{9(n+4)}{6}+\frac{18(4 n-2)}{9}+\frac{(n-2)}{12} \\
& =2(7 n-2),
\end{aligned}
$$


$(c)$

$$
\begin{aligned}
A Z I(\Gamma) & =\left.S_{x}^{3} J\left(D_{x}^{3}\left(D_{y}^{3}(f(x, y))\right)\right)\right|_{x=1=y} \\
& =\left(\frac{9(n+4)}{4}\right)^{3}+\left(\frac{18(4 n-2)}{7}\right)^{3}+\left(\frac{36(n-2)}{10}\right)^{3} \\
& =\frac{345903939}{2744000} n-\frac{56084157}{686000} .
\end{aligned}
$$

Theorem 3.7. Jlabelt3.7 Let $\Gamma=O X(n)$ be the oxide network. Then, the $M$-polynomial of $\Gamma$ is

$$
M(\Gamma, x, y)=12 n x^{2} y^{4}+\left(18 n^{2}-12 n\right) x^{4} y^{4} .
$$

Proof. From Figure 3, we note that there are two types of vertices in $\Gamma$ with respect to their degree such as of degree 2 and 4 , and three types of edges with respect to degree of end vertices, that is, $\{2,2\}$ and $\{4,4\}$. Thus, we have

$$
V_{1}=\{u \in V(\Gamma) \mid d(u)=2\} \quad \text { and } \quad V_{2}=\{u \in V(\Gamma) \mid d(u)=4\}
$$

with $\left|V_{1}\right|=6 n$, and $\left|V_{2}\right|=9 n^{2}-3 n$, respectively. Consequently, $|V(\Gamma)|=$ $9 n^{2}+3 n$. Similarly, we have

$$
\begin{aligned}
& E_{1}=E_{2,4}=\{u v \in E(\Gamma) \mid d(u)=2, d(u)=4\}, \\
& E_{2}=E_{4,4}=\{u v \in E(\Gamma) \mid d(u)=4, d(u)=4\}
\end{aligned}
$$

such that $\left|E_{1}\right|=12 n$ and $\left|E_{2}\right|=18 n^{2}-12 n$. We conclude $|E(\Gamma)|=18 n^{2}$. Thus, the partitions of the vertex set and the edge set of the oxide network are given in the Tables 7 and 8 .

Table 7. The partitions of the vertex set of the oxide network $O X(n)$

\begin{tabular}{|c|c|c|}
\hline Vertex partition & $V_{1}$ & $V_{2}$ \\
\hline Cardinality & $6 n$ & $9 n^{2}-3 n$ \\
\hline
\end{tabular}

Table 8 . The partitions of the edge set of the oxide network $O X(n)$

\begin{tabular}{|c|c|c|}
\hline Edge partition & $E_{1}=E_{2,4}$ & $E_{2}=E_{4,4}$ \\
\hline Cardinality & $12 n$ & $18 n^{2}-12 n$ \\
\hline
\end{tabular}


Now, by the use of Definition 2.6 and the Tables 7 and 8, $M$-polynomial of $\Gamma$ is

$$
\begin{aligned}
M(\Gamma, x, y) & =\sum_{i \leq j}\left[E_{i, j}(\Gamma) x^{i} y^{j}\right] \\
& =\sum_{2 \leq 4}\left[E_{2,4}(\Gamma) x^{2} y^{4}\right]+\sum_{4 \leq 4}\left[E_{4,4}(\Gamma) x^{4} y^{4}\right] \\
& =\left|E_{1}\right| x^{2} y^{4}+\left|E_{2}\right| x^{4} y^{4} \\
& =12 n x^{2} y^{4}+\left(18 n^{2}-12 n\right) x^{4} y^{4}
\end{aligned}
$$

Theorem 3.8. Let $\Gamma=O X(n)$ be the oxide network and

$$
M(\Gamma, x, y)=12 n x^{2} y^{4}+\left(18 n^{2}-12 n\right) x^{4} y^{4}
$$

be its $M$-polynomial. Then, the first Zagreb index $\left(M_{1}(\Gamma)\right)$, the second Zagreb index $\left(M_{2}(\Gamma)\right)$, the second modified Zagreb $\left(M M_{2}(\Gamma)\right)$, general Randić, $\left(R_{\alpha}(\Gamma)\right)$, where $\alpha \in \mathbb{N}$, reciprocal general Randić, $\left(R R_{\alpha}(\Gamma)\right)$, where $\alpha \in \mathbb{N}$ and the symmetric division degree index $(S D D(\Gamma))$ obtained from $M$-polynomial are as follows:
(s) $M_{1}(\Gamma)=24 n(6 n-1)$,
(b) $M_{2}(\Gamma)=96 n(3 n-1)$,
(c) $M M_{2}(\Gamma)=\frac{3}{8}\left[3 n^{2}+2 n\right]$,
(d) $R_{\alpha}(\Gamma)=3(2)^{3 \alpha+1}\left[3(2)^{\alpha} n^{2}+2\left(1-2^{\alpha}\right) n\right]$,
(e) $R R_{\alpha}(\Gamma)=\frac{3}{2^{4 \alpha-1}}\left[3 n^{2}+2\left(2^{\alpha}-1\right) n\right]$,
(f) $S D D(\Gamma)=6 n(6 n+1)$.

Proof. Let $f(x, y)=M(\Gamma, x, y)$ be the $M$-polynomial of the oxide network. Then

$$
f(x, y)=12 n x^{2} y^{4}+\left(18 n^{2}-12 n\right) x^{4} y^{4} .
$$

Now, the partial derivatives and integrals are obtained as

$$
\begin{aligned}
& D_{x}(f(x, y))=24 n x y^{4}+4\left(18 n^{2}-12 n\right) x^{3} y^{4}, \\
& D_{y}(f(x, y))=48 n x^{2} y^{3}+4\left(18 n^{2}-12 n\right) x^{4} y^{3}, \\
& D_{x}\left(D_{y}(f(x, y))\right)=96 x y^{3}+16\left(18 n^{2}-12 n\right) x^{3} y^{3}, \\
& S_{x}(f(x, y))=6 n x^{2} y^{4}+\frac{9 n^{2}-6 n}{2} x^{4} y^{4} \\
& S_{y}(f(x, y))=3 n x^{2} y^{4}+\frac{9 n^{2}-6 n}{2} x^{4} y^{4} \\
& S_{x} S_{y}(f(x, y))=\frac{3 n}{2} x^{2} y^{4}+\frac{9 n^{2}-6 n}{8} x^{4} y^{4}, \\
& D_{y} S_{x}(f(x, y))=24 n x^{2} y^{3}+\left(18 n^{2}-12 n\right) x^{4} y^{3}, \\
& D_{x} S_{y}(f(x, y))=6 n x y^{4}+\left(18 n^{2}-12 n\right) x^{3} y^{4},
\end{aligned}
$$




$$
\begin{aligned}
& D_{x}^{\alpha}\left(D_{y}^{\alpha}(f(x, y))\right)=\left(8^{\alpha}\right)(12 n) x y^{3}+\left(16^{\alpha}\right)\left(18 n^{2}-12 n\right) x^{3} y^{3}, \\
& S_{x}^{\alpha} S_{y}^{\alpha}(f(x, y))=\frac{12 n}{8^{\alpha}} x^{2} y^{4}+\frac{18 n^{2}-12 n}{16^{\alpha}} x^{4} y^{4} .
\end{aligned}
$$

Now, we obtain

$$
\begin{aligned}
& \left.D_{x}(f(x, y))\right|_{x=1=y}=72 n^{2}-24 n, \\
& \left.D_{y}(f(x, y))\right|_{x=1=y}=12 n^{2}, \\
& \left.D_{x}\left(D_{y}(f(x, y))\right)\right|_{x=1=y}=288 n^{2}-96 n, \\
& \left.S_{x}(f(x, y))\right|_{x=1=y}=6 n+\frac{9 n^{2}-6 n}{2}, \\
& \left.S_{y}(f(x, y))\right|_{x=1=y}=\frac{9}{2} n^{2}, \\
& \left.S_{x} S_{y}(f(x, y))\right|_{x=1=y}=\frac{3}{2} n+\frac{9 n^{2}-6 n}{8}, \\
& \left.D_{y} S_{x}(f(x, y))\right|_{x=1=y}=18 n^{2}+12 n \\
& \left.D_{x} S_{y}(f(x, y))\right|_{x=1=y}=18 n^{2}-6 n, \\
& \left.D_{x}^{\alpha}\left(D_{y}^{\alpha}(f(x, y))\right)\right|_{x=1=y}=\left(8^{\alpha}\right)(12 n)+\left(16^{\alpha}\right)\left(18 n^{2}-12 n\right), \\
& \left.S_{x}^{\alpha} S_{y}^{\alpha}(f(x, y))\right|_{x=1=y}=\frac{12 n}{8^{\alpha}}+\frac{18 n^{2}-12 n}{16^{\alpha}} .
\end{aligned}
$$

Consequently,

(a)

$$
\begin{aligned}
M_{1}(\Gamma) & =\left.\left(D_{x}+D_{y}\right)(f(x, y))\right|_{x=1=y} \\
& =\left.D_{x}(f(x, y))\right|_{x=1=y}+\left.D_{y}(f(x, y))\right|_{x=1=y} \\
& =\left(72 n^{2}-24 n\right)+\left(72 n^{2}\right)=24 n(6 n-1),
\end{aligned}
$$

(b)

$$
\begin{aligned}
M_{2}(\Gamma) & =\left.\left(D_{x} D_{y}\right)(f(x, y))\right|_{x=1=y} \\
& =\left.D_{x}\left(D_{y}(f(x, y))\right)\right|_{x=1=y} \\
& =288 n^{2}-96 n=96 n(3 n-1),
\end{aligned}
$$

(c)

$$
\begin{aligned}
M M_{2}(\Gamma) & =\left.\left(S_{x} S_{y}\right)(f(x, y))\right|_{x=1=y} \\
& =\left.S_{x}\left(S_{y}(f(x, y))\right)\right|_{x=1=y} \\
& =\frac{3}{2} n+\frac{9 n^{2}-6 n}{8}=\frac{3}{8}\left[3 n^{2}+2 n\right],
\end{aligned}
$$

(d)

$$
\begin{aligned}
R_{\alpha}(\Gamma) & =\left.\left(D_{x}^{\alpha} D_{y}^{\alpha}\right)(f(x, y))\right|_{x=1=y} \\
& =\left(8^{\alpha}\right)(12 n)+\left(16^{\alpha}\right)\left(18 n^{2}-12 n\right) \\
& =3(2)^{3 \alpha+1}\left[3(2)^{\alpha} n^{2}+2\left(1-2^{\alpha}\right) n\right]
\end{aligned}
$$


(e)

$$
\begin{aligned}
R R_{\alpha}(\Gamma) & =\left.\left(S_{x}^{\alpha} S_{y}^{\alpha}\right)(f(x, y))\right|_{x=1=y} \\
& =\left(8^{\alpha}\right)(12 n) x y^{3}+\left(16^{\alpha}\right)\left(18 n^{2}-12 n\right) x^{3} y^{3} \\
& =\frac{3}{2^{4 \alpha-1}}\left[3 n^{2}+2\left(2^{\alpha}-1\right) n\right]
\end{aligned}
$$

$(f)$

$$
\begin{aligned}
S D D(\Gamma) & =\left(D_{x} S_{y}+D_{y} S_{x}\right)(f(x, y)) \\
& =\left(D_{x} S_{y}\right)(f(x, y))+\left(D_{y} S_{x}\right)(f(x, y)) \\
& =D_{x}\left(S_{y}(f(x, y))\right)+D_{y}\left(S_{x}(f(x, y))\right) \\
& =\left(18 n^{2}+12 n\right)+\left(18 n^{2}-6 n\right) \\
& =6 n(6 n+1) .
\end{aligned}
$$

Theorem 3.9. Let $\Gamma=O X(n)$ be the oxide network and

$$
M(\Gamma, x, y)=12 n x^{2} y^{4}+\left(18 n^{2}-12 n\right) x^{4} y^{4}
$$

be its $M$-polynomial. Then, harmonic index $(H(\Gamma))$, inverse sum index $(I S(\Gamma))$, and augmented Zagreb index $(A Z I(\Gamma))$ obtained from $M$-polynomial are as follows:

(a) $H(\Gamma)=\frac{n}{2}[9 n+2]$,

(b) $I S(\Gamma)=4 n(9 n-2)$,

(c) $A Z I(\Gamma)=\frac{1024}{3} n^{2}-\frac{1184}{9} n$.

Proof. Let $f(x, y)=M(\Gamma, x, y)$ be the $M$-polynomial of the oxide network. Then

$$
f(x, y)=12 n x^{2} y^{4}+\left(18 n^{2}-12 n\right) x^{4} y^{4} .
$$

Now, the required expressions are obtained as

$$
\begin{aligned}
& J(f(x, y))=12 n x^{6}+\left(18 n^{2}-12 n\right) x^{8}, \\
& S_{x}(J f(x, y))=2 n x^{6}+\frac{9 n^{2}-6 n}{4} x^{8}, \\
& J\left(D_{x}\left(D_{y}(f(x, y))\right)\right)=96 n x^{4}+16\left(18 n^{2}-12 n\right) x^{6}, \\
& Q_{2} J\left(D_{x}\left(D_{y}(f(x, y))\right)\right)=96 n x^{6}+16\left(18 n^{2}-12 n\right) x^{8}, \\
& S_{x} Q_{2} J\left(D_{x}\left(D_{y}(f(x, y))\right)\right)=16 n x^{6}+2\left(18 n^{2}-12 n\right) x^{8}, \\
& D_{x}^{3}\left(D_{y}^{3}(f(x, y))\right)=\left(8^{3}\right)(12 n) x^{4}+\left(16^{3}\right)\left(18 n^{2}-12 n\right) x^{6}, \\
& J D_{x}^{3}\left(D_{y}^{3}(f(x, y))\right)=\left(8^{3}\right)(12 n) x^{4}+\left(16^{3}\right)\left(18 n^{2}-12 n\right) x^{6}, \\
& S_{x}^{3} J\left(D_{x}^{3}\left(D_{y}^{3}(f(x, y))\right)\right)=\frac{8^{3}(12 n)}{4^{3}} x^{4}+\frac{16^{3}\left(18 n^{2}-12 n\right)}{6^{3}} x^{6} . \\
& \text { Now, we obtain }\left.S_{x}(J f(x, y))\right|_{x=1=y}=2 n+\frac{9 n^{2}-6 n}{4},
\end{aligned}
$$


$\left.S_{x} Q_{2} J\left(D_{x}\left(D_{y}(f(x, y))\right)\right)\right|_{x=1=y}=16 n+2\left(18 n^{2}-12 n\right)$,
$\left.S_{x}^{3} J\left(D_{x}^{3}\left(D_{y}^{3}(f(x, y))\right)\right)\right|_{x=1=y}=\frac{8^{3}(12 n)}{4^{3}}+\frac{16^{3}\left(18 n^{2}-12 n\right)}{6^{3}} .$.

Consequently,

(a)

$$
\begin{aligned}
H(\Gamma) & =\left.2 S_{x}(J f(x, y))\right|_{x=1=y} \\
& =2\left[2 n+\frac{9 n^{2}-6 n}{4}\right]=\frac{n}{2}[9 n+2],
\end{aligned}
$$

(b)

$$
\begin{aligned}
I S(\Gamma) & =\left.S_{x} Q_{2} J\left(D_{x}\left(D_{y}(f(x, y))\right)\right)\right|_{x=1=y} \\
& =16 n+2\left(18 n^{2}-12 n\right)=4 n(9 n-2),
\end{aligned}
$$

(c)

$$
\begin{aligned}
A Z I(\Gamma) & =\left.S_{x}^{3} J\left(D_{x}^{3}\left(D_{y}^{3}(f(x, y))\right)\right)\right|_{x=1=y} \\
& =\frac{8^{3}(12 n)}{4^{3}}+\frac{16^{3}\left(18 n^{2}-12 n\right)}{6^{3}} \\
& =\frac{1024}{3} n^{2}-\frac{1184}{9} n .
\end{aligned}
$$

\section{Conclusions}

In this paper, we proved the $M$-polynomials of the silicate, chain silicate and oxide networks. With the help of these $M$-polynomials, we also computed the certain degree-based topological indices such as first Zagreb, second Zagreb, second modified Zagreb, general Randić, reciprocal general Randic, symmetric division deg, harmonic index, inverse sum index and the augmented Zagreb index of these networks. In other words, we can say the $M$-polynomials are used to compute the certain degree based topological indices as a latest developed tool in the chemical graph theory.

Moreover, the obtained $M$-polynomials and all the computed topological indices are expressed in terms of $n$, where $n$ shows the dimension of the each network studied in this note. Figures 4, 5 and 6 show the $A Z I$ as a better one for each network studied in the current paper.

Now, we close our discussion with the following lines. These counting polynomials and computed topological indices can help us to understand the physical features, chemical reactivity and biological activities of the silicate, chain 
silicate and oxide networks. These results can also provide a significant determination in the pharmaceutical industry $[13,23]$.

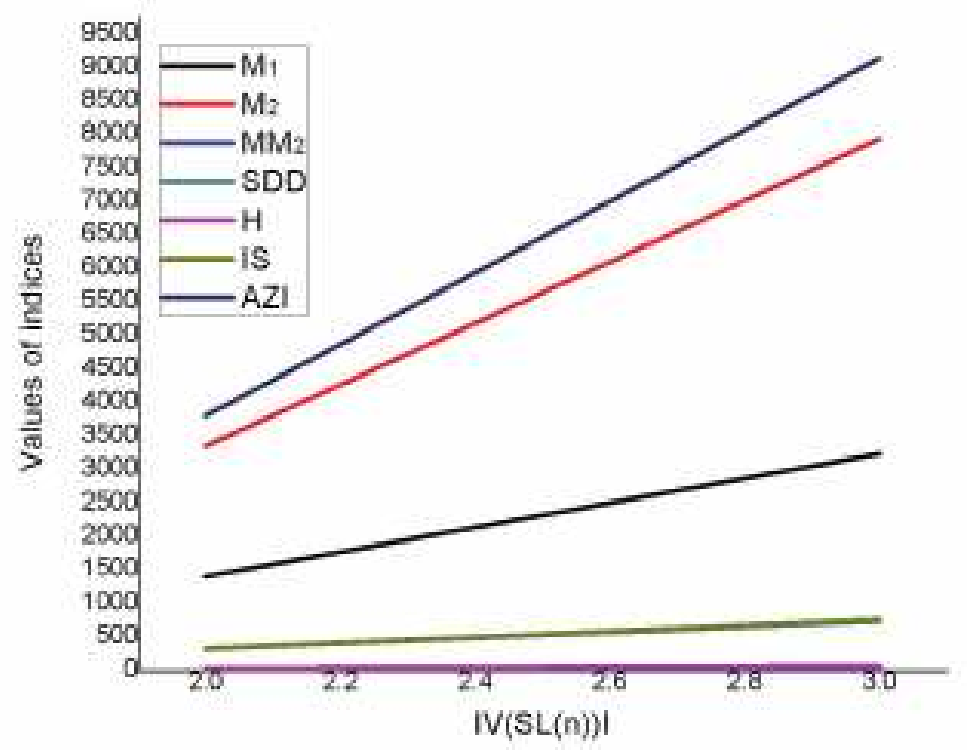

Figure 4. Comparison of the computed indices of $S L(n)$ 


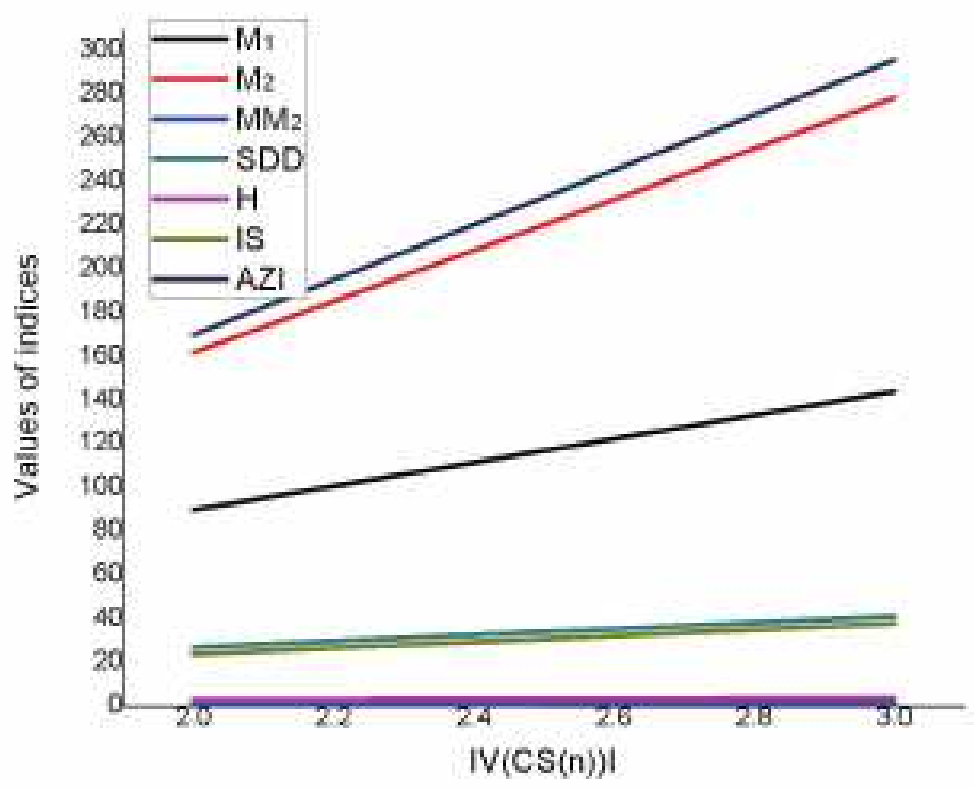

Figure 5. Comparison of the computed indices of $C S(n)$.

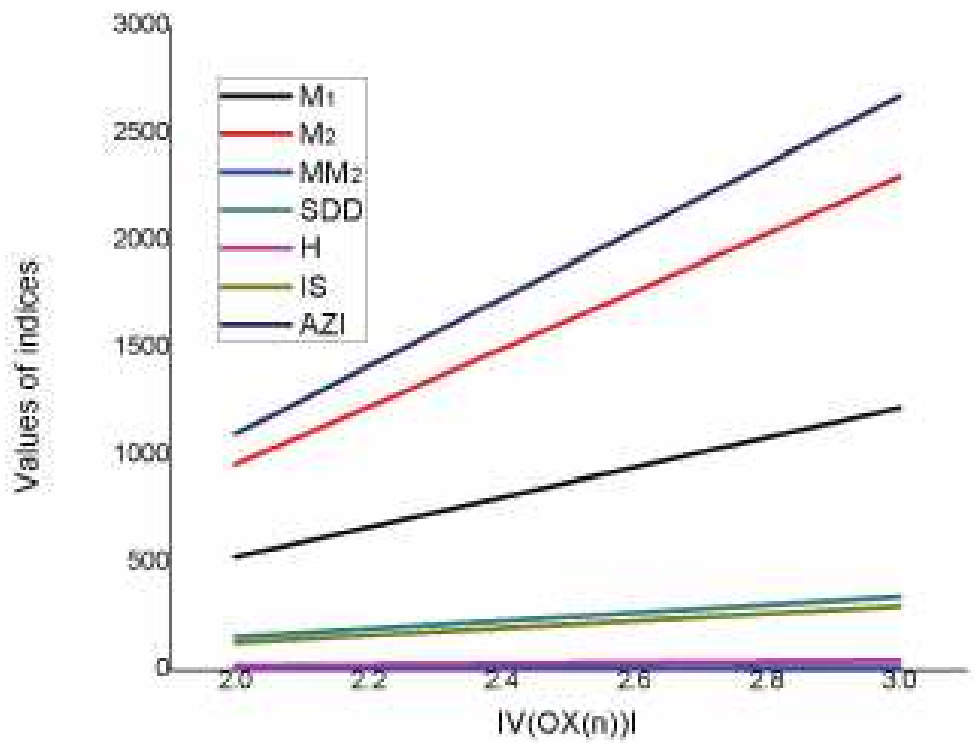

Figure 6. Comparison of the computed indices of $O X(n)$. 


\section{References}

[1] D. Amić, D. Bešlo, B. Lučić, S. Nikolić, N. Trinajstić, The vertex-connectivity index revisited, J. Chem. Inf. Comput. Sci., 38 (1998), 819-822, doi: 10.1021/ci980039b.

[2] M. Bača, J. Horváthová, M. Mokrišová, A. Semaničová-Feňovčková, A. Suhányiová, On topological indices of a carbon nanotube network, Canad. J. Chem. 93 (2015), 1157-1160, doi: $10.1139 /$ cjc-2015-0175.

[3] M. Bača, J. Horváthová, M. Mokrišová, A. Suhányiová, On topological indices of fullerenes, Appl. Math. Comput., 251 (2015), 154-161, doi: 10.1016/j.amc.2014.11.069.

[4] B. Bollobás, P. Erdös, Graphs of extremal weights, Ars Combin., 50 (1998), 225-233.

[5] F.M. Brückler, T. Došlić, A. Graovac, I. Gutman, On a class of distance-based molecular structure descriptors, Chem. Phys. Lett., 503 (2011), 336-338, doi: 10.1016/j.cplett.2011.01.033.

[6] E. Deutsch, S. Klavžar, $M$-polynomial and degree-based topological indices, Iran. J. Math. Chem., 6 (2015), 93-102, doi: 10.22052/ijmc.2015.10106.

[7] J. Devillers, A.T. Balaban, Topological Indices and Related Descriptors in QSAR and QSPR, Gordon and Breach, Amsterdam, 1999.

[8] M.V. Diudea, QSPR/QSAR Studies by Molecular Descriptors, Nova Science Publ. Inc, New York, 2000.

[9] B. Furtula, A. Graovac, D. Vukičević, Augmented Zagreb index, J. Math. Chem., 48 (2010), 370-380, doi: 10.1007/s10910-010-9677-3.

[10] I. Gutman, Degree-based topological indices, Croat. Chem. Acta, 86 (2013), 351-361, doi: $10.5562 /$ cca2294.

[11] I. Gutman and O. Polansky, Mathematical Concepts in Organic Chemistry, SpringerVerlag, Berlin, 1986.

[12] I. Gutman and N. Trinajstić, Graph theory and molecular orbitals. Total $\pi$-electron energy of alternant hydrocarbons, Chem. Phys. Lett., 17 (1972), 535-538, doi: 10.1016/0009-2614(72)85099-1.

[13] L.H. Hall, L.B. Kier, Molecular Connectivity in Chemistry and Drug Research, Academic Press, Boston, 1976.

[14] F. Harary, Graph Theory, Addison-Wesley Publishing Co., 1969.

[15] M. Javaid, M.U. Rehman, J. Cao, Topological indices of rhombus type silicate and oxide networks, Canan. J. Chem., 95 (2017), 134-143, doi: 10.1139/cjc-2016-0486.

[16] S. Klavžar, I. Gutman, A Comparison of the Schultz molecular topological index with the Wiener index, J. Chem. Inf. Comput. Sci., 36 (1996), 1001-1003, doi: 10.1021/ci9603689.

[17] M.A. Malik, M. Imran, On multiple Zagreb indices of $\mathrm{TiO}_{2}$ nanotubes, Acta Chim. Slov., 62 (2015), 973-976, doi: 10.17344/acsi.2015.1746.

[18] M. Munir, W. Nazeer, Z. Shahzadi, S.M. Kang, $M$-polynomial and degree-based topological indices of polyhex nanotubes, Symmetry, 8 (2016), Article ID 149, 8 pages, doi: 10.3390/sym8120149.

[19] G. Pólya, Kombinatorische Anzahlbestimmungen für Gruppen, Graphen und chemische Verbindungen, Acta Math., 68 (1936), 145-253, doi: 10.1007/BF02546665. 
[20] B. Rajan, A. William, C. Grigorious, S. Stephen, On certain topological indices of silicate, honeycomb and hexagonal networks, J. Comp. Math. Sci., 3 (2012), 530-535.

[21] M. Randić, On characterization of molecular branching, J. Amer. Chem. Soc. 97 (1975), 6609-6615, doi: 10.1021/ja00856a001.

[22] G. Rucker, C. Rucker, On topological indices, boiling points, and cycloalkanes, J. Chem. Inf. Comput. Sci., 39 (1999), 788-802, doi: 10.1021/ci9900175.

[23] R. Todeschini, V. Consonni, Handbook of Molecular Descriptors, Wiley-VCH Verlag, Weinheim, Germany, 2000.

[24] D.B. West, Introduction to Graph Theory, Printce Hall, Inc., New Jersery, 1996.

[25] H.J. Wiener, Structural determination of paraffin boiling points, J. Amer. Chem. Soc., 69 (1947), 17-20, doi: 10.1021/ja01193a005. 